\title{
QUENCH PROTECTION FOR SUPERCONDUCTING IR MAGNETS IN CESR*
}

\author{
G. Codner $^{+}$, G. Dugan, W. Lou ${ }^{\#}$, J. Welch \\ Laboratory of Nuclear Studies, Cornell University, Ithaca, NY 14853
}

\begin{abstract}
Analysis and design details are provided on quench protection for the Cornell Electron Storage Ring (CESR) Phase III interaction region (IR) superconducting magnets. The proposed scheme extracts much of the stored energy thereby reducing thermal stress on the magnets and mitigating liquid helium loss. Protection is somewhat complicated by the fact that each cold mass consists of quadrupole, skew quadrupole and dipole windings, and that each cryostat contains two magnet units.
\end{abstract}

\section{INTRODUCTION}

The CESR (Cornell Electron Storage Ring) Phase III Upgrade includes a low beta interaction region that includes several superconducting (SC) magnets [1]. There are two (2) magnet assemblies comprised of two (2) magnet units per assembly. Each unit includes a main quadrupole, a skew quadrupole and a dipole magnet. The three magnets in each unit are integrally wound into as small a space as possible. This paper describes quench detection and protection for these magnets.

\section{APPROACH}

The CESR SC IR magnets are designed to survive quenches without damage, even without active protection. However, it is prudent to minimize stress on the magnets by detecting quenches and dumping as much of the energy as is practical into an external load. Furthermore, diverting this energy reduces the amount of liquid helium that would be boiled off in the event of a quench, thereby saving time and money. Since it is not necessary to divert all of the stored energy, the simple approach of using a fast, electro-mechanical D.C. switch is used.

CESR quench detection uses the coil voltage comparison method. Since the voltage across a winding that is quenching is relatively low (the voltage across the quench resistance is mostly canceled by $L d I / d t$ of the coil), the rate of change of current must be detected, assuming the current regulation loop is slow. The simplest way to do this is to sense the voltage across another coil in the same magnet. Assuming both windings do not quench simultaneously, the changing current due to the quench induces a voltage across the nonquenching coil.

*Work supported by the National Science Foundation

+email gcodner@Ins.cornell.edu

"Now with ADC Telecommunications, Shelton, CT

\subsection{Calculations}

Fundamental calculations were performed [2] to determine the quench resistance as a function of time, the relationship between deposited energy and coil temperature and to find the optimal value of the dump resistor $R_{d}$ for each magnet. A trade-off must be made between extracted energy and magnet lead voltage. $R_{d}$ was chosen such that the maximum internal and external voltages during a quench are equal.

For the CESR SC IR magnets, a normal zone bounded in two dimensions after a time $t_{a}$ is applicable. The magnet's time-dependent resistance during the early part of a quench may be roughly approximated by [3]:

$$
R_{Q}(t)=\left\{\begin{array}{ll}
\frac{3 L t^{5}}{t_{Q}^{6}} \quad \text { for } 0 \leq t<t_{a} \\
\frac{L\left(15 t_{a}^{2} t^{3}-15 t_{a}^{3} t^{2}+3 t_{a}^{5}\right)}{t_{Q}^{6}} & \text { for } t \geq t_{a}
\end{array}\right\}
$$

where $t_{a}$ and $L$ are parameters determined by the physical characteristics of each magnet coil whereas $t_{Q}$ depends on the physical magnet parameters and the initial stored energy. Table 1 lists currently predicted or measured values of these parameters for the three types of magnets.

Table 1: Calculated CESR SC IR Magnet Parameters

\begin{tabular}{||ccccc||}
\hline $\begin{array}{c}\text { Magnet } \\
\text { Type }\end{array}$ & $\begin{array}{c}\mathbf{t}_{\mathbf{a}} \\
{[\mathbf{s e c}]}\end{array}$ & $\begin{array}{c}\mathbf{t}_{\mathbf{Q}} \\
{[\mathbf{s e c}]}\end{array}$ & $\begin{array}{c}\mathbf{L}^{*} \\
{[\mathbf{h e n r y}]}\end{array}$ & $\begin{array}{c}\mathbf{I}_{\mathbf{o}} \\
\text { [amps] }\end{array}$ \\
\hline Main Quad & 0.067 & 0.45 & 0.244 & 1225 \\
Skew Quad & 0.024 & 0.12 & 0.115 & 325 \\
Dipole & 0.047 & 0.18 & 0.024 & 200 \\
\hline
\end{tabular}

*measured

\subsection{Spice Modeling}

A circuit model was used to explore the behavior of the detection method and to perform the calculations done in [2] based on the latest information available including measured magnet data, the actual detection circuitry and time delay of the quench switch. These parameters were used in a PSpice model that simulates the growth in quench resistance with time and the action of the detection circuitry and quench switch. The model may also include power supply behavior, for example, fast current regulation. 


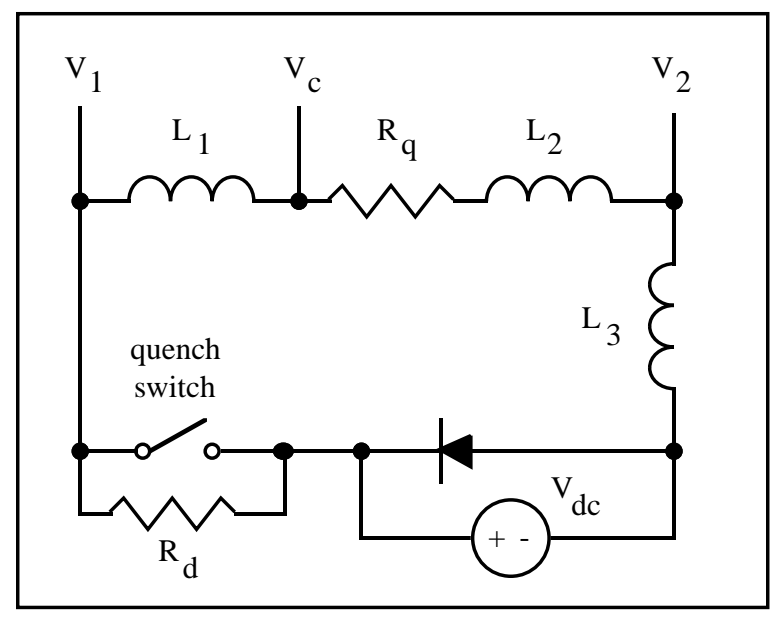

Figure 1: Equivalent circuit of CESR SC IR magnet and quench protection for a quench in coil $L_{2}$ (the quench switch is shown in its protection state).

The PSpice model includes the effects of coupling between windings as opposed to a simpler model that considers the total inductance to be split into equal parts for each winding. It was found that coupling was not very important in its effect on quench detection and that the simple model is adequate for these calculations.

Figure 1 shows an equivalent circuit of a magnet during a quench. $L_{1}$ and $L_{2}$ are coils that are sensed for comparison. A quench in $L_{2}$ is represented by timedependent resistor $R_{q}$. The quench switch opens after a quench is detected placing $R_{d}$ in series with the magnet. In the simple model $L_{3}$ represents any other coils in series with the magnet, for example, the other two coils in a quadrupole magnet. The model with coupling uses each coil explicitly.

The standard method of sensing coil voltages uses a common point between two coils. However, a quench that starts at this common point would propagate in both directions away from that point so in that case this method of detection would fail or produce a long delay. Therefore, we will compare voltages of opposite facing coils of the quads. The dipole is still susceptible to some quenches that would not be detected in a timely manner, however, the stored energy is low and the consequences of delayed quench detection in that case are minimal.

\subsection{Calculation Results}

Table 2 lists dump resistor values and quench protection delay times. Figure 2 shows the current waveform for the main quad with a total delay time of 0.19 second due to

Table 2: Dump resistor values and protection times

\begin{tabular}{||ccc||}
\hline $\begin{array}{c}\text { Magnet } \\
\text { Type }\end{array}$ & $\begin{array}{c}\text { Dump } \\
\text { Resistor } \\
\text { [ohm] }\end{array}$ & $\begin{array}{c}\text { Protection } \\
\text { Delay } \\
\text { [seconds] }\end{array}$ \\
\hline Main Quad & 0.24 & 0.19 \\
Skew Quad & 0.70 & 0.13 \\
Dipole & 0.11 & 0.16 \\
\hline
\end{tabular}

the chosen detection threshold, quench propagation time and quench switch actuation time of $100 \mathrm{~ms}$. Table 3 lists calculated results for the three types of magnets.

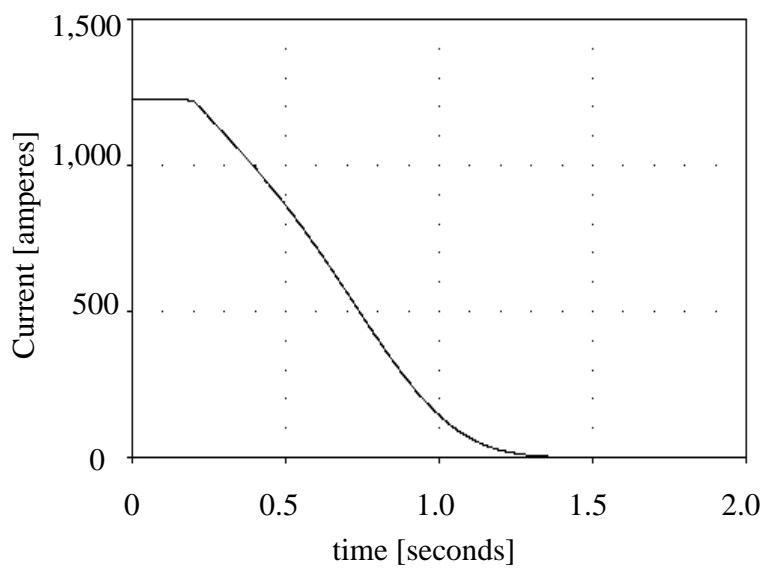

Figure 2: CESR SC IR main quad predicted current.

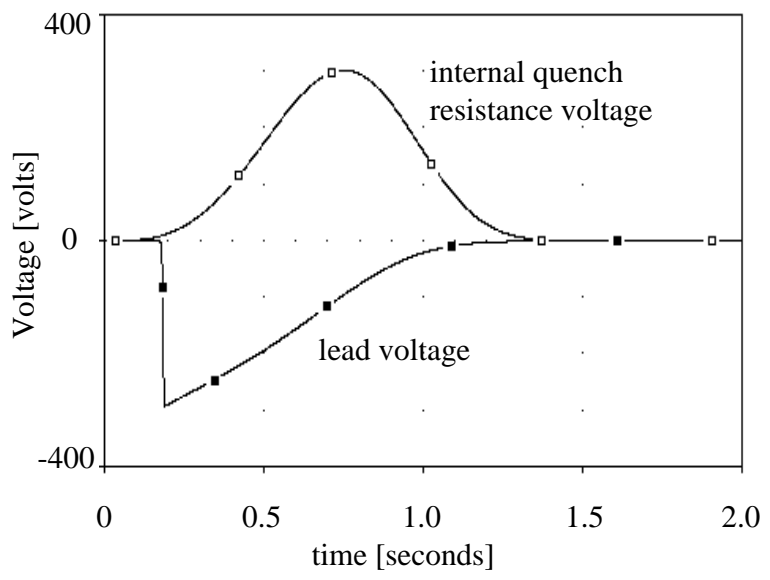

Figure 3: CESR SC IR main quad predicted voltages

Table 3: Calculated energy and voltage

\begin{tabular}{||cccc||}
\hline $\begin{array}{c}\text { Magnet } \\
\text { Type }\end{array}$ & $\begin{array}{c}\text { Stored } \\
\text { Energy } \\
{[\mathbf{k J}]}\end{array}$ & $\begin{array}{c}\text { Extracted } \\
\text { Energy } \\
{[\mathbf{k J}]}\end{array}$ & $\begin{array}{c}\text { Peak } \\
\text { Voltage } \\
\text { [volts] }\end{array}$ \\
\hline Main Quad & 183 & 107 & 300 \\
Skew Quad & 6.1 & 1.9 & 198 \\
Dipole & 0.48 & 0.15 & 19 \\
\hline
\end{tabular}

\section{DESIGN}

Figure 4 is a block diagram of the quench detection and protection system. There are two magnet assemblies in CESR, one in the East IR and one in the West IR. Each magnet assembly contains two units, each unit comprising a main quad, a skew quad and a dipole magnet. Each magnet has leads brought out from superconducting regions for sensing magnet coil voltages. With the sense leads attached to superconducting regions and with little current flow in these leads, the desired 0.5 volt detection threshold is reasonably high. For the main quad, this threshold gives a detection delay time approximately equal to the quench switch delay time. 


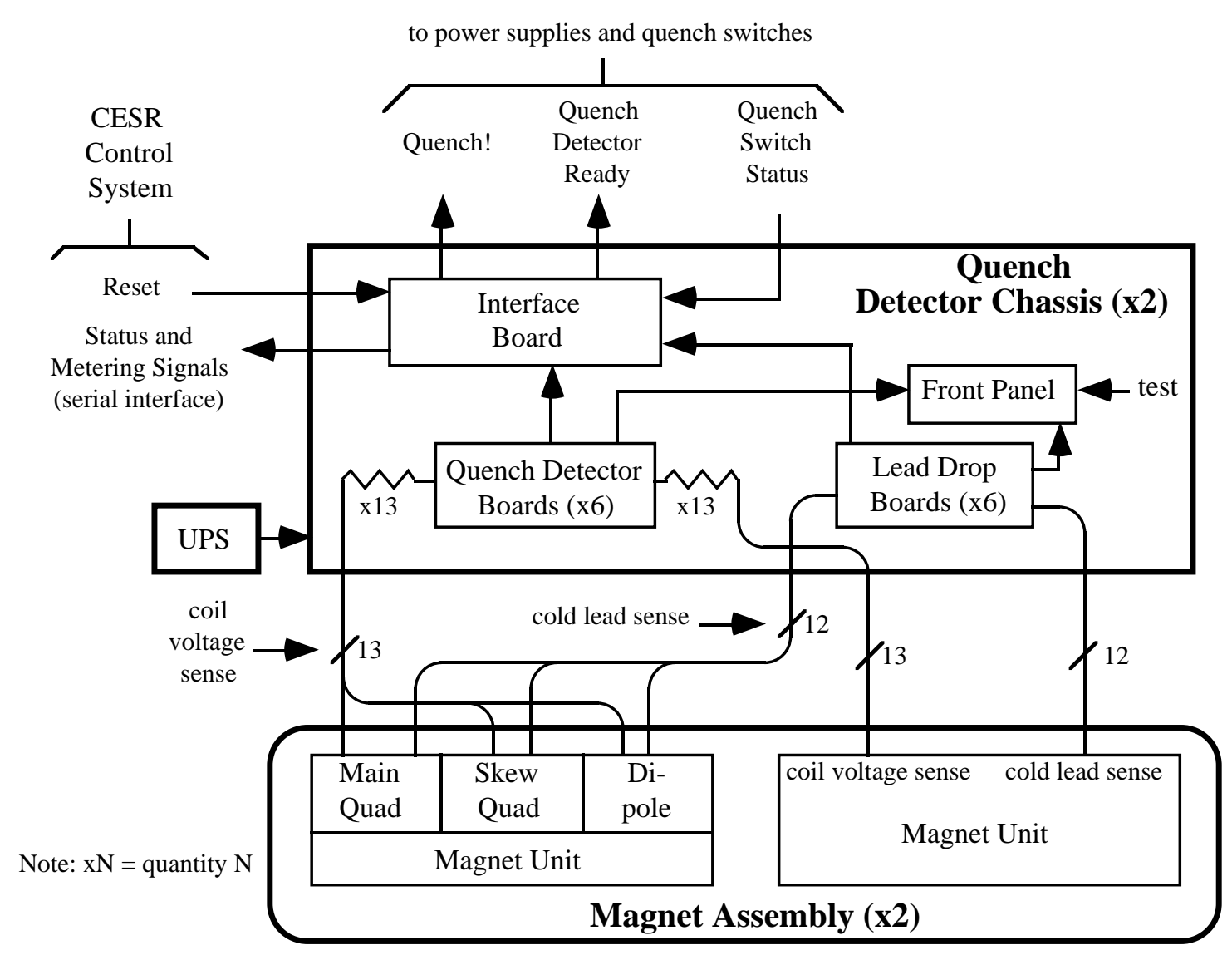

Figure 4: CESR Superconducting Interaction Region Magnet Quench Detection System.

The coil sense leads enter the quench detection chassis through connectors rated at 600 volts. Current limiting resistors are placed in series with each lead which then connect to isolation amplifiers on the detector boards. The AD202 isolation amplifier has a common mode range of \pm 1000 volts, but a maximum differential voltage of only \pm 5 volts so the input must be clamped.

Appropriate coil voltages are subtracted and applied to window comparators with \pm 0.5 volt thresholds. The comparator outputs are latched until reset by the CESR control system. A quench detected in one magnet of a unit opens the quench switches for all three magnets in that unit and also turns off the power supplies for all six magnets associated with that side of the IR.

Electrically actuated D.C. contactors are used as quench protection switches and stamped, stainless steel resistors are used for dump resistors. There is one quench switch and one dump resistor per magnet.

To reduce the number of nuisance quench detections, the quench detector chassis are powered from a common Uninterruptible Power Supply (UPS) allowing the system to operate through line transients and power outages.

Each quench detection chassis also houses monitoring electronics for the vapor-cooled magnet leads. Excess voltage drop in a lead activates the quench switches to protect the magnet leads from over dissipation.

\section{CONCLUSION}

An approach for quench detection and protection of CESR superconducting interaction region magnets has been described. A design has been presented based on the predicted behavior of these magnets. When the magnets are fully tested, actual coil voltages and currents during quenches will be measured to test the calculations presented here.

\section{ACKNOWLEDGEMENTS}

Thanks to Mike Comfort, Ted Vandermark and Ron Yaeger at Wilson Lab for their expertise and to the National Superconducting Cyclotron Laboratory at Michigan State University.

\section{REFERENCES}

[1] J.J. Welch, G. Dugan, E. Nordberg, D. Rice, "The Superconducting Interaction Region Magnet System for the CESR Phase III Upgrade", Cornell University, PAC97

[2] G. Dugan, "Quench Protection Considerations for CESR Superconducting IR Quadrupoles," CBN 97-27, http://www.lns.cornell.edu/public/CBN/1997/.

[3] M.N. Wilson, Superconducting Magnets, p. 214, Oxford:1983. 